\title{
DESCRIPTION OF HYDRO/METEO DATA IN SHIP MANOEUVRING SIMULATORS: A SURVEY ON THE STATE OF THE ART
}

\author{
Luca Donatini and Marc Vantorre, Maritime Technology Division, Ghent University, Belgium \\ Jeroen Verwilligen, Flanders Hydraulics Research, Belgium \\ Guillaume Delefortrie, Flanders Hydraulics Research, Belgium and Maritime Technology Division, Ghent University, \\ Belgium
}

\section{SUMMARY}

This paper describes the results of a survey performed by the authors to assess how hydro/meteo conditions are presently modelled in ship manoeuvring simulators. A questionnaire regarding current, waves, wind and water levels was sent to a selected list of simulator developers and users. The received answers were thoroughly analysed and are summarized in this paper as an overview of the state of the art in hydro/meteo data for ship simulators at the time of writing. All the results are published in an anonymous form to guarantee a high discretion level to all parties who answered the survey.

\section{INTRODUCTION}

Ship manoeuvring simulators are nowadays regarded as invaluable tools in both the design of port infrastructures and the training of maritime professionals. The level of realism of simulations increased dramatically in the last decade due to the ever increasing availability of computational power impacting on both mathematical modelling and visual performances.

Manoeuvring simulation software is in a permanent state of development, for different reasons. The evolution of computer hardware nowadays allows to perform calculations in real-time which were absolutely impossible a few decades ago. On the other hand, customers have increasing demands with respect to the complexity of simulations, the realism of both ship behaviour and environmental conditions, and the accuracy and reliability of the results. Because of improved position measurement systems, operations are performed with decreasing margins which also means that both mathematical models for ship manoeuvring and the representation of the meteorological and hydrological conditions have to meet higher standards.

While mathematical models for the manoeuvring behaviour of ships are well documented in literature, an overview concerning hydro/meteo modelling in ship manoeuvring simulators is hard to find in the public domain. In order to determine a strategy for future developments in this respect, Flanders Hydraulics Research (FHR) has commissioned the Maritime Technology Division of Ghent University (UGent) to perform a study to determine an optimal way of representing waves, currents, tides and wind. In the frame of such a study, it is appropriate to examine the state of the art, not only by studying the specialized literature, but also by contacts with other developers, scientists and advanced users who are involved in similar matters. As such, not only a better view will be obtained on the common practice, but exchange of ideas might also lead to a better communication and even co-operation.

In order to investigate the current state of art of hydro/meteo modelling in ship simulators, a questionnaire was sent to a selected list of simulator developers and users. The explicit aim of the questionnaire was the collection of information to be publicly summarized in the form of a scientific publication. The answers from several parties which took the effort to return a filled in questionnaire were collected and analysed, and are summarized in this paper. All the received data are published here in an anonymous way to ensure the appropriate level of confidentiality to all the parties involved.

The information summarized in this paper could benefit the whole community of ship simulator users and developers by making everyone more aware of the present common practices, while also fixing a starting point for future research and improvements.

\section{QUESTIONNAIRE}

The questionnaire on hydro/meteo modelling was developed by UGent in collaboration with FHR with the aim to collect information about the way current, waves, wind and water levels are presently modelled in ship manoeuvring simulators worldwide. The questionnaire was sent to approximately thirty parties, selected between simulator developers and advanced users. Twelve replies were received in time to be included in this paper.

The questionnaire contains five main sections, investigating different aspects of hydro/meteo modelling:

1. General information

2. Current

3. Waves

4. Wind

5. Water levels

Each of these different sections will be analysed in more detail in a following chapter.

\section{GENERAL INFORMATION}

The general information section of the questionnaire contains questions about the replying institute and the types of simulators in use. The twelve received replies come from ten different countries, and can be categorized as in the following. 
Two replies came from universities, four from public research institutes and six from private companies. For convenience, all will be generally referred as "institutes" in the paper. Ten of the replies came from simulator developing institutes and two from simulator users. All of the developer institutes except for one are involved in the development of both the mathematical model and the visual part of simulators. All the developer institutes use their own simulators for design consultancy purposes, eight of them also use simulators for scientific research and five among this eight also for training purposes. On the other hand, the two simulator users mainly use simulators for design/consultancy purposes, with only one of them using simulators also for scientific research.

All replying institutes develop or use simulators based on 6 DOF mathematical models. Eleven institutes deal with full mission bridge simulators, while one deals with a part task simulator, composed of a reduced set of nautical instruments and visualization devices. One of the institutes only deals with inland navigation simulations, while the others are mainly focused on confined, coastal or open water simulations.
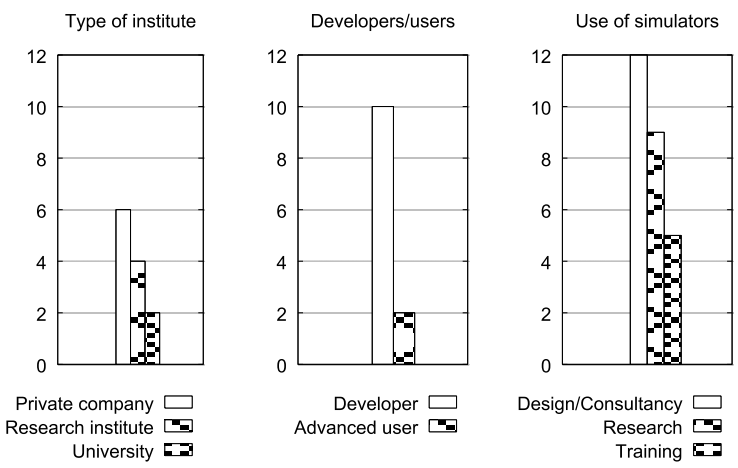

Figure 1. General information.

\section{CURRENT}

Current fields are present in almost all the environments where a ship operates, due to different physical phenomena. At open sea, surface oceanic currents are induced principally by wind and Coriolis effects. Near the coast, on the other hand, currents are mainly induced by the tidal excursion and by river outlets, due to both the river discharge and the associated density flow. More locally, currents can also be originated by the effects of man-made constructions like locks, weirs and harbours. Examples of such effects are discharges due to lock openings and tidal induced currents at harbour entrances. Due to the ubiquitous presence of current and to the relevant effect it has on the manoeuvring behaviour of ships, it is a very significant parameter to be modelled in order to improve the realism of simulations.

\subsection{CURRENT FIELD REPRESENTATION}

The questions concerning current field representation investigate how the current field and its space and time variability are modelled in simulators.

Current is represented as a $2 \mathrm{D}$ vector of horizontal speed by most of the institutes who participated to the survey. Only two institutes out of twelve use a more advanced 3D vector representation of current which includes a vertical speed component.

A spatial variation of the current field is modelled by most of the survey participants: only one does not foresee the possibility to deal with a variable current field in the horizontal plane. Among the institutes that deal with horizontal variations of the current field, ten implement the possibility of variable grid size, while the remaining one can only manage a fixed grid size on the whole domain. Concerning the interpolation of gridded current field values in the horizontal direction, seven repliers use linear interpolation, one uses upwind interpolation and three use proprietary algorithms which were not disclosed. The horizontal resolution of the current fields is dependent on several different factors, like the type of application or the resolution of the numerical models providing the results (when used). According to the received replies the horizontal resolution for the current field used in simulators is on average between $20 \mathrm{~m}$ and $100 \mathrm{~m}$. As notable exceptions, one of the replying institutes can go as low as a couple of meters in coastal areas and as high as $500 \mathrm{~m}$ in offshore areas, while another institute uses a default resolution of less than one meter.

The vertical variation of current fields is completely neglected by six repliers. Among the remaining six institutes, four consider such variation in the preprocessing phase, by calculating a depth averaged current field and using this 2D field as the input for the simulator. In these cases, the depth average can be performed based either on the water depth or on the vessel's draft. The depth averaging process can be based on a simple average of the current velocity or on an average of the velocity squared, which leads to more significant averaged values from the point of view of the forces and moments generated by current. The remaining two institutes use a fully $3 \mathrm{D}$ current field as a direct input to simulators. One of these two institutes actually implements a depthaveraging procedure in real time during the simulation. For each time step, a draft averaged current velocity is calculated for each of the 2D longitudinal strips in which the ship is discretized. The draft averaging is based on the velocity squared. The last institute discretizes the ship by means of a voxel approach, with a small number of depth layers (usually 3 ). The current velocity acting on each voxel is calculated through an interpolation of the input 3D current field based on a proprietary algorithm.

Among all six institutes which consider vertical variations of the current field, either in the form of depth averaged or fully 3D current fields, two use an absolute vertical coordinate system, while four can choose between an absolute system and a relative coordinate system based on the local and temporal water depth. 
Concerning time variation of the current field, eight replying institutes use this feature, one institute does not use the feature even if it is available and three institutes do not provide this functionality. The time intervals for current field updates range between less than a second and a couple of hours. In the last case, a sine function is fitted to the current field values available at the update times. Five institutes adopt linear interpolation in time between the input current fields, two use not disclosed proprietary interpolation algorithms and one does not interpolate.
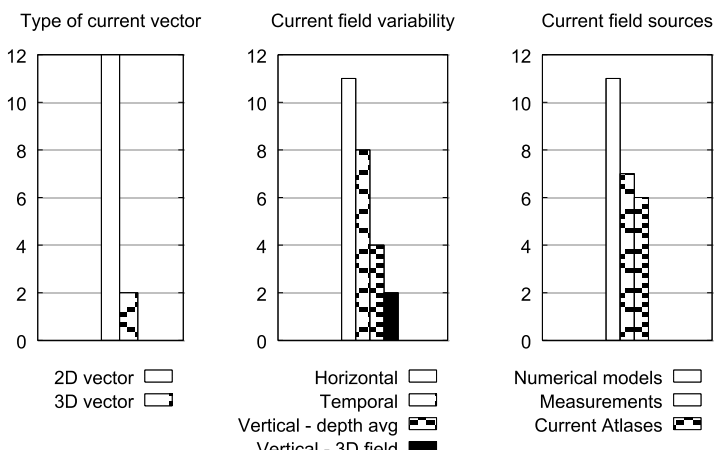

Figure 2. Current field representation.

\subsection{SOURCE AND FORMAT OF CURRENT INPUTS}

The next sub-section of the questionnaire investigates the sources of current field data and the input file formats. Eleven institutes out of twelve have the possibility to get current fields data from dedicated simulations performed with hydraulic software. Only one institute does not foresee this option, while for another one it is the only possible one. Different numerical models are used by different institutes, usually depending on in-house availability or client requests. Seven institutes can directly use current field data coming from measurements, and six can use current atlases and databases. However, at least two institutes pointed out that current measurements, while not being a direct input for the simulator, have a crucial role in the validation and calibration of numerical results.

Concerning the format of input files for current fields, it is strictly dependent on the simulator software. The picture coming out from the survey is that there is no recognized standard: proprietary file formats, simple ASCII files, xml files and MATLAB files are some of the possibilities according to the received answers. Most institutes write converters to convert current fields in arbitrary formats to the format which their simulator expects. Two of the replying institutes are currently investigating the possibility to adopt netCDF as an exchange file format. NetCDF is a binary format commonly used in atmospheric and oceanographic simulations to store large amounts of array-oriented data in an organized and self-describing way.

\subsection{CURRENT INDUCED FORCES AND MOMENTS}

The questionnaire focuses then on how current fields are used to calculate hydrodynamic forces and moments acting on the ship. First of all, the number of degrees of freedom which the current effects are accounted for is investigated. Two of the replying institutes consider current effects only in 2DOF: surge and sway. Six institutes consider the current effects in $3 \mathrm{DOF}$, taking also into account yaw. Two institutes consider the effects in $4 \mathrm{DOF}$, adding roll to the picture. Finally, two institutes considers current effects in all 6DOF. One of these two institutes specifies that, due to the modelling of current as a 2D vector (no vertical speed component), the current effects in heave and pitch are due to current induced squat motions.

The most common way to calculate current induced forces and moments, adopted by ten of the replying institutes, is to calculate the mean current speeds and accelerations acting on the vessel in each of the considered DOF. When a horizontal variation of the current field is considered, the mean current speeds are calculated as an average of the sectional current speeds which are interpolated in real time at different positions along the ship length. In the reply where the vertical variation of current is also taken into account, the sectional speeds are in turn obtained by means of a draft averaging procedure for each 2D strip (see previous paragraph). The mean current speed components acting on the hull are combined with the vessel's own speed components to obtain relative speed through water components, which are fed into the manoeuvring model. The current effects on the hull are therefore accounted for by using relative speed components to calculate the hydrodynamic forces and moments. One institute adopts a different approach: the ship is discretized in 20 sections, and current induced forces and moments are calculated for each section using a sectional drag coefficient, which is assumed to be constant along the hull. The contribution of the different sections is integrated along the ship length to produce the total current induced force and moment. Finally, the institute which implements fully 3D current fields models the hull through a voxel approach and calculates current induced pressures acting on each voxel based on Bernoulli equation. The voxel pressures are then integrated to provide current induced forces and moments. 

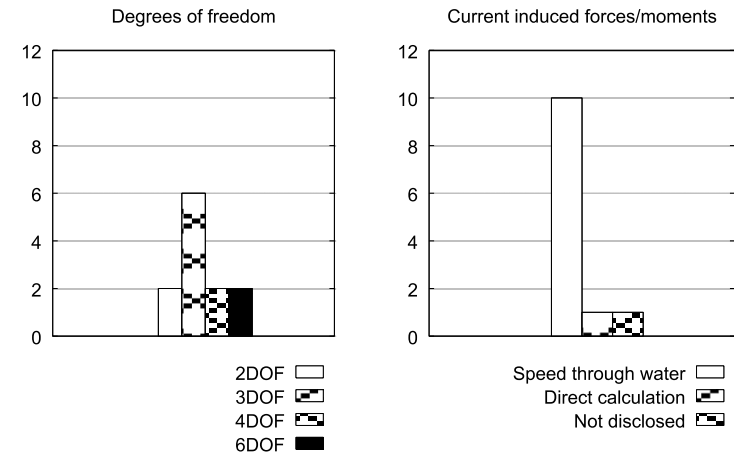

Figure 3. Current induced forces and moments.

\subsection{ADDITIONAL FEATURES}

Additional current features investigated in the questionnaire are the effects of density stratified fluids and the influence of the ship's presence on the current field in confined water.

Concerning the effects of stratified fluids, eleven of the replying institutes do not consider them, while one points out that such effects are taken into account by the hydraulic models which provide the current field input.

Concerning the effects of the ship on the current field in confined water, only two institutes replied that their simulators are capable of accounting for such an effect. In one case, an additional speed is added to the current speed to represent the speed increase under the hull of the ship due to Venturi effects. Only the longitudinal component of the current velocity is considered in the process. In the other case, the way this effect is accounted for is not disclosed. Among the ten institutes which do not include the effects of the ship on the current field in confined water, seven describe such an effect as highly desirable feature.

\section{WAVES}

Among the different types of gravity waves occurring on the oceans, wind generated waves are the ones which have the highest impact on ships. In order to be generated, wind waves need two factors: a forcing wind field and a sufficiently large water extension, called fetch, over which the wind can blow. Wind waves are in fact generated locally as very short ripples and can evolve into long waves along the fetch due to the wind which keeps transferring energy to the generated waves. When analysing a local wave climate, a distinction is usually made between the wind sea, which refers to waves generated in place by the local wind field, and swell, which refers to waves generated far away which have propagated to the location of interest.

Wave effects are generally not present in inland waterways, rivers or harbours. When dealing with simulations in these environments, wave effects can usually be neglected. An important exception to this consideration needs to be raised when the conditions occur for internal wave agitation phenomena. When an enclosed basin has reflective boundaries and an open inlet, the external sea waves can penetrate in the basin and keep evolving inside it for a long time due to repeated reflections on the boundaries. The magnitude of this physical process is strongly dependent on both the precise harbour geometry and the detailed characteristics of forcing waves. In certain conditions, the effects of internal wave agitation can be relevant for the ship behaviour.

Wave effects on a ship can be crudely split into two: second order wave effects, which induce mean drift forces acting on time scales comparable to the ones characterizing the manoeuvring dynamics, and first order wave effects, which induce oscillatory motions at higher frequencies. This distinction is at the base of the two time scales approach to the modelling of ship manoeuvring behaviour in waves. According to this approach, the wave induced drift loads influence the manoeuvrability behaviour, and are therefore of major relevance for a ship manoeuvring simulator; the oscillatory motions, on the other hand, are considered independent and are optionally included in simulators mainly to improve realism. In this case, wave effects can be dealt with in the frequency domain resorting to wave spectra and response amplitude operators (RAOs).

A different approach to the problem is based on a unified description of the hydrodynamic problem. This requires a more elaborated mathematical model, where wave effects need to be accounted for in the time domain.

\subsection{WAVE FIELD REPRESENTATION}

The next section of the questionnaire investigates the description of waves in ship manoeuvring simulators. All replying institutes apart from two include the effect of waves. One of the institutes neglecting waves deals only with inland navigation while the another one deals mainly with confined water and inland navigation. The majority of institutes which include wave effects, nine, model waves in the form of wave spectra. Only one institute adopts a different approach, implementing an internal wave model based on wind inputs and a simplified fetch based formulation. Among the nine institutes which adopt a spectral representation, all can work with unidirectional, frequency dependent wave spectra, and six of them can also work with directional wave spectra. The institute adopting an internal simplified wave model can model different wave systems by setting up different input winds coming from different directions and with different fetches.

Six of the nine institutes dealing with wave spectra take into account a spatial variability of such quantity. The temporal variability of wave spectra, on the other hand, is neglected by almost all repliers. This can be ascribed to the fact that the time scales of significant changes in wave spectra, usually between 30 minutes and a few hours, are longer than the common duration of a real time simulation. Therefore, the wave climate can be considered as steady during real time simulations. One institute does not disclose information concerning spatial or temporal variations of wave spectra. Among the six institutes 
implementing a spatial variability of the wave spectra, three do not interpolate wave data in space, one performs linear interpolation of the wave spectra and two perform linear interpolation of integral parameters like the significant wave height and mean wave direction, reconstructing the directional spectrum based on theoretical spectral formulations and directional spreading functions.

Five of the replying institutes can use the results of wave agitation models (Bousinnesq, mild-slope, ...) for describing the wave climate in restricted waters.

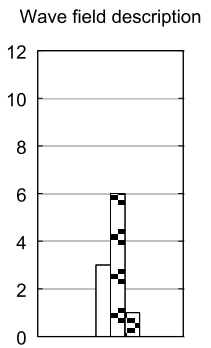

Freq. spectrum $\square$ Dir. spectrum $\square$ Fetch model

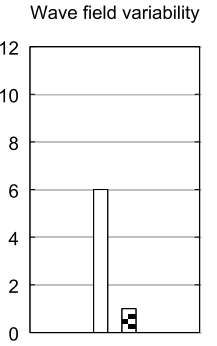

Space $\square$ Temporal $\square$ Not disclosed

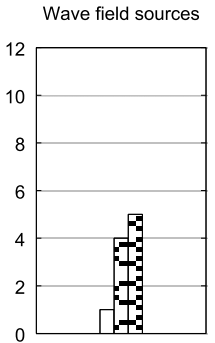

User defined wind $\square$ ser defined waves $\square$ Measurements/models

\section{Figure 4. Wave field description.}

\subsection{SOURCE AND FORMAT OF WAVE INPUTS}

Concerning the source of wave data to be used in simulations, three main options were outlined from the received answers: wave measurements, numerical wave models and user defined wave parameters. Five institutes rely mainly on user defined parameters. One of these institutes is the one implementing a simplified fetch based wave model: in this case, the user defined parameters are wind speed, direction and fetch length. For the other four institutes relying on user defined parameters, these are classic integral wave parameters such as significant wave height, mean wave direction and wave period. Such parameters are converted into a spectral representation by the use of theoretical wave spectrums (e.g. JONSWAP, Bretschneider, ...) and directional spreading functions (e.g. $\left.\cos 2, \cos -2 \mathrm{~s}^{1}, \ldots\right)$.

The remaining five institutes can take wave input data either from wave buoy measurements or from the results of wave models calibrated with measured data coming from buoys or satellites. In most cases, only integral data are obtained from wave models or buoys, and theoretical spectra are fitted to the input wave data. In at least one case, a bi-modal spectrum approach is used, which distinguishes between wind sea and swell. A JONSWAP spectrum describes the wind sea, coupled with a cos-2s spreading function, while the swell is modelled through a uni-directional JONSWAP spectrum (larger peakedness gamma factor) or through a regular wave.

${ }^{1}$ Widely used distribution where the s parameter accounts for the spreading of wave energy around a mean wave direction.
No general indications about common formats for input wave data were found from the received answers.

\subsection{WAVE INDUCED FORCES AND MOMENTS}

Among the ten institutes which implement waves in simulations, nine consider the wave induced effects on the ship in all 6 degrees of freedom, while one only considers the effects in 4 DOF: surge, sway, roll and pitch. Concerning the wave effects took into consideration, all ten institutes consider $1^{\text {st }}$ order wave induced oscillatory motions as well as $2^{\text {nd }}$ order mean wave drift forces in the horizontal plane. In one case, first order motions are calculated in 3 DOF (roll, pitch and heave) while second order drift forces and moments are considered in the remaining 3 DOF (surge, sway and yaw). Five institutes also consider time-varying $2^{\text {nd }}$ order wave forces (slowlyvarying) in the horizontal plane.

Nine institutes have the option to use Response Amplitude Operators (RAOs) to calculate oscillatory ship motions in the frequency domain. Frequency domain motions are usually transformed in the time domain by means of Fourier transforms. Two of these nine institutes have the alternative possibility to directly calculate wave exciting forces and radiation forces in the time domain and to solve the equations of motion for first order oscillatory motions in real time. This approach allows to consider non-linear Froude-Krylov forces and to achieve a tighter integration between the manoeuvring and seakeeping mathematical models. One of the institutes does not have the option to use RAOs due to wave modelling approach: this is the institute which models waves through an internal fetch based wave model (see previous paragraph), and therefore does not consider wave spectra. In this case, wave forces and moments are calculated only by an integration of non-linear FroudeKrylov forces calculated on the ship hull, which is discretized by means of a voxel approach. None of the replying institutes resorts to an external physical engine for the calculation of wave induced forces and moments.

Seven of the ten institutes which implement waves describe a direct correlation between the wave pattern used in the mathematical model and the one used in the visualization part. As for the three exceptions, in one case the match between the two wave patterns is not exact: the same integral parameters (significant wave height, mean wave direction and wave period) are used, but the directional spreading for the visualization part is calculated independently from the one used in the mathematical model. One institute distinguishes between swell and wind sea: for swell there is a direct correlation between the visuals and the mathematical model, while for wind sea the two representations are independent. In the mathematical model wind sea is represented as a spectrum, while in the visualization part it is modelled through an external visualization engine based on the local 
wind speed. Finally, one institute reports no direct correlation between the two wave patterns.
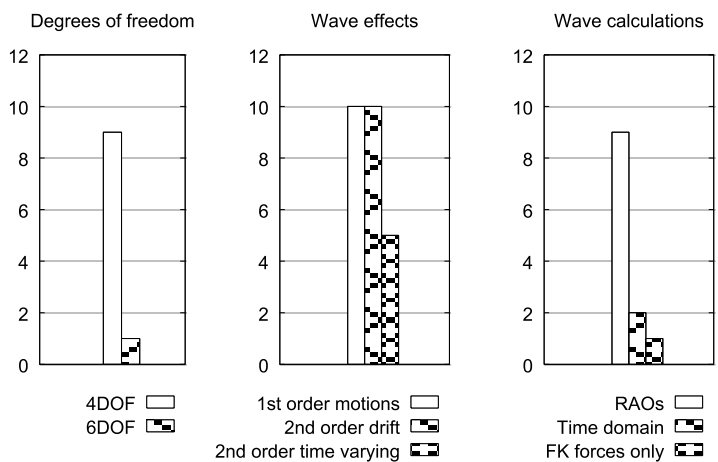

Figure 5. Wave induced forces and moments.

\subsection{CURRENT-WAVE INTERACTION}

The only additional wave modelling feature investigated in the questionnaire concerns the interaction between current and waves. Three institutes reply that this effect is taken into account in the calculation of wave induced motions. One of them specifies that this is done through the calculation of a wave drift damping which takes into account the influence of waves on drift forces. Another institute points out that the effect of current on the wave field is accounted for in the numerical wave models providing wave data input to the simulator. The last institute does not provide additional details about how this feature is implemented. One institute does not disclose if this feature is implemented or not.

\section{WIND}

As for current, a ship can be subject to wind in all its operating environments. Wind acts on the upper works of a ship, mainly originating a force in the horizontal plane and a yawing moment. Due to the vertical distance between the point of application of the wind induced force and the point of application of the resisting hydrodynamic force, a heeling moment is also originated. The resulting heeling angle, which can be large, changes the geometry of the hull, and therefore also the manoeuvring behaviour of the ship. The effects of wind on the ship manoeuvring behaviour can be relevant, especially for ships with tall cargo and/or superstructures, like container vessels, cruise ships and car carriers.

\subsection{WIND FIELD REPRESENTATION}

Concerning the representation of wind fields in simulators, first of all the questionnaire focuses on turbulent fluctuations of wind speed and direction in time. Two of the replying institutes assume a constant wind speed, neglecting turbulence fluctuations. Two institutes only consider fluctuations in the wind speed, while all the other eight institutes model turbulent fluctuations of both wind speed and direction.
The questionnaire then investigates the variability of the mean wind fields in space and time. Three institutes do not take the horizontal variability of mean wind fields into account, and model a constant mean wind field in space. This is independent from the modelling of turbulent fluctuations described above: despite considering a uniform mean wind field over the simulation domain, two of the institutes which do not model a spatial variability still take into account turbulent wind fluctuations. The other nine institutes take spatial variability of wind fields into consideration. One of them only does so based on the sheltering effects induced by terrain elevation, buildings or other vessels. No additional details concerning typical grid sizes or spatial interpolation techniques for wind fields were made available through the received answers. Concerning the vertical variation of wind fields, only two institutes take it into account, by implementing a vertical wind distribution which affects only the wind speed magnitude. A vertical variation of the wind field is accounted for also by another institute, but only with respect to the effects of wind sheltering by land features or other vessels.

Six institutes out of twelve can model time variations of the mean wind field along a simulation. Ten of the replying institutes, on the other hand, foresee the possibility for the simulator operator to adapt the wind fields in real time.

\subsection{SOURCE AND FORMAT OF WIND INPUTS}

A clear picture about the source of input wind fields used in simulators could not be obtained from the received replies. When constant wind speed and direction are assumed over the simulation domain, their values are usually selected by the simulator operator based on the desired environmental conditions and on experience about the local wind climate of the area to be simulated. On the other hand, when a spatial variability of the mean wind field is foreseen, the spatial distribution is most likely the result of a numerical model. At least two of the replying institutes have the possibility to use wind fields calculated by CFD codes and converted to $2 \mathrm{D}$ fields as the input for simulations. The details for such a conversion are not disclosed.

No indications can be derived from the received answers about specific file formats used for providing input wind fields to simulators. 

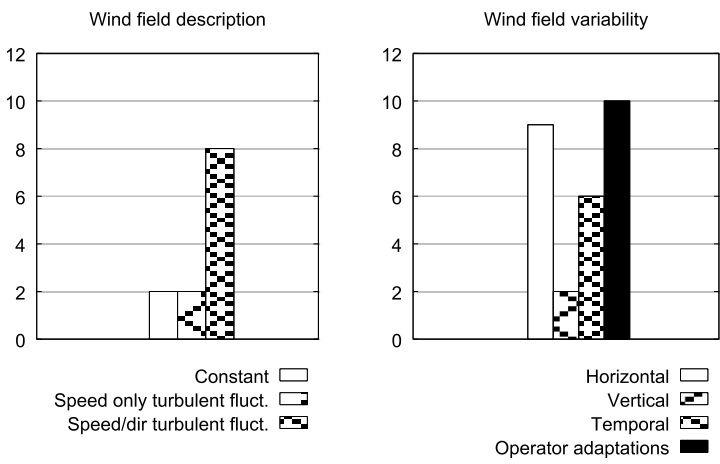

Figure 6. Wind field description.

\subsection{WIND INDUCED FORCES AND MOMENTS}

Six of the replying institutes model wind induced forces and moments in 4 degrees of freedom: surge, sway, yaw and roll (heel). Four institutes model wind effects in the horizontal 3 DOF, while two institutes model the effects in all $6 \mathrm{DOF}$.

Nine institutes take into account the horizontal variation of wind speed along the ship length in the calculation of wind induced forces and moments. In at least two cases this is done by interpolating the input wind field at a number of points along the ship length and then calculating an average wind vector. The relative wind vector (taking into account the ship's motion) is then combined with wind coefficients to provide aerodynamic forces and moments. In another case, the ship superstructure is modelled through a voxel approach, and the force exerted by the wind field on each voxel is integrated over the whole superstructure. No additional information was obtained from the remaining three institutes. Two of the replying institutes also take into account the vertical variability of the input wind field in the calculation of wind induced forces and moments.

Only three institutes consider the sheltering effect of the ship on the input wind field. In one case, the input wind field is modified inside a box which travels with the ship and is reshaped according to the incident wind speed and direction. No information is disclosed by the other two institutes. Among the institutes which do not consider the sheltering effect of the ship on the input wind field, at least three point out that this is a highly desired feature and a topic for further developments.
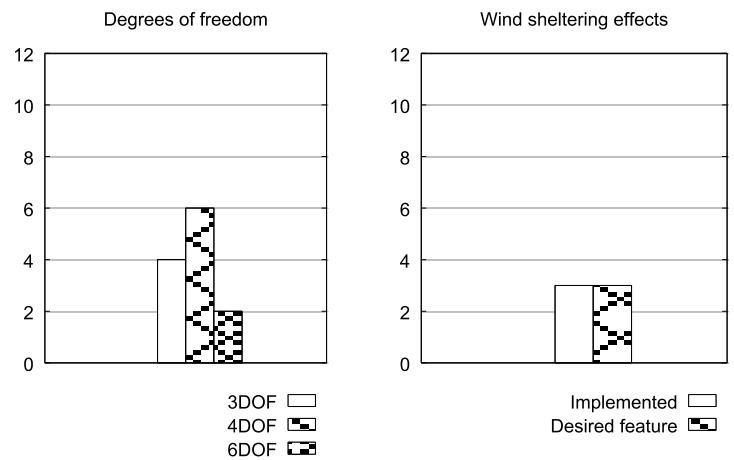

Figure 7. Wind induced forces and moments.

\section{WATER LEVELS}

Ocean water levels change due to tidal effects. The magnitude of tidal excursion depends on the geographic location, and is usually higher near to the coast line. Water levels can also change in man-made hydraulic structures such as locks. Due to the large periods of water level changes, a ship does not experience significant forces due to such changes. Therefore, in sufficiently deep water, water level changes have no effect on the manoeuvring behaviour of a ship. However, when the water depth is comparable with the ship's draft, the ship's manoeuvring behaviour is significantly influenced by the under keel clearance (UKC), which is defined as the ratio between the vertical distance from the keel line to the sea bottom and the draft of the vessel. Due to this, the effects of changing water levels need to be taken into account in order to achieve realistic manoeuvring simulations in shallow water.

\subsection{WATER LEVELS REPRESENTATION}

Regarding the representation of water levels in simulations, two institutes only considers a spatial and temporal constant value for the whole simulation. Nine institutes consider a temporal variation of the water level along the simulation, while five consider spatial variations of the water level over the simulation domain. Four of the institutes which consider a temporal variation also consider spatial variations, while one considers spatial variations only. Four institutes reflect the spatial variations of water levels in the visuals, for example in the evident case of two sides of a lock.

\subsection{SOURCE AND FORMAT OF WATER LEVEL INPUTS}

Similarly to what happened for wind input, no clear picture can be drawn concerning the source of water levels input. Usually, this information either comes from the same numerical models which provide current field inputs or is set up by simulator operators based on their experience.

Apart from one institute resorting to netCDF (see also 4.2) as the input file format for water levels, no other 
information can be derived from the received answers concerning this topic as well.

\subsection{ROLE OF WATER LEVELS IN MATHEMATICAL MODEL}

The final section of the questionnaire investigates how water levels are accounted for in the mathematical models of simulators. Nine of the replying institutes model the effects of water levels by using different mathematical models for predefined under keel clearance (UKC) values. Usually, the water level at the ship position is interpolated from the input water levels, if needed, and a UKC value is calculated based on the ship's draft. The forces acting on the ship are then calculated according to the mathematical model corresponding to the calculated UKC. One institute adopts a different approach, based on a single mathematical model and direct calculations of corrections to the hydrodynamic forces in confined water. The remaining two institutes did not specify how water levels are taken into account.
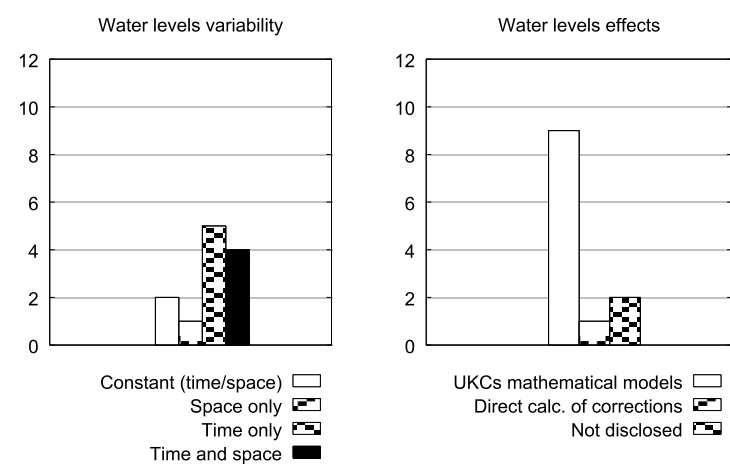

Figure 8. Water levels description and effects.

\section{CLOSING REMARKS}

Ship manoeuvring simulations have proven to be of great utility for a wide list of applications, which range from the design of port infrastructures to the training of maritime professionals. The usefulness of simulations is tightly linked with the degree of accuracy and realism which can be attained. Current, wind, waves and water levels can all have a large impact on the behaviour of ships. Therefore, the realism of simulations does not only depend on the accuracy of the mathematical models which describe the manoeuvring behaviour of ships, but also, to a great extent, on how the hydro/meteo conditions and their effects on ships are modelled. While mathematical models for the manoeuvring behaviour of ships have been and still are largely discussed in scientific literature, the modelling of hydro/meteo conditions lacks a publicly available reference literature. With the aim to start filling this gap, a survey on the state of the art of hydro/meteo conditions modelling in ship manoeuvring simulators was developed by the authors. A questionnaire focusing on current, waves, wind and water levels was sent to approximately 30 parties, selected between simulators developers and advanced users. Among the recipients, only 12 institutes replied to the survey in time for their answers to be included in this work. The reluctance of a large part of the contacted parties to provide answers on the topic reveals that a description of the implementation of hydro/meteo conditions in a simulator is not considered as an objectively technical and scientific information. While this can be partially understood in the logic of commercial competition, it also prevents the crucial exchange of information needed to push forward the boundaries of simulation accuracy. The results of the survey published in this paper outline some common approaches as well as some consistent differences in how hydro/meteo conditions and their effects on ship behaviour are modelled by different institutes. In the hope of the authors, the present work represents a first step towards a more scientific approach to the modelling of hydro/meteo conditions in ship manoeuvring simulators, and also a possible starting point for collaborations aimed at the common goal to make ship manoeuvring simulations ever more realistic.

\section{ACKNOWLEDGEMENTS}

The present work is performed in the frame of project WL 201706 (Scientific support for the implementation of seakeeping in the ship manoeuvring simulators), granted to Ghent University by Flanders Hydraulics Research, Antwerp (Department of Mobility and Public Works, Flemish Government, Belgium).

The authors wish to thank all institutes who participated to the questionnaire.

\section{AUTHORS BIOGRAPHY}

Luca Donatini, naval architect, is a $\mathrm{PhD}$ student at Ghent University. He is currently working on a project aimed at improving the modelling of hydro/meteo effects in the ship manoeuvring simulator of Flanders Hydraulic Research. His previous experiences encompass seakeeping studies to allow inland vessels on a Belgian sea trajectory and the inclusion of hydro/meteo effects in an open source mooring dynamics code. He also has an extensive experience in atmospheric and spectral wave modelling from global to regional scales.

Marc Vantorre, naval architect, is emeritus professor of marine hydrodynamics and former head of the Maritime Technology Division at Ghent University, Belgium. His research focuses on ship behaviour in shallow and confined waters, mainly in close co-operation with Flanders Hydraulics Research in Antwerp. He is former member of PIANC Working Groups and of the ITTC Manoeuvring Committee..

Jeroen Verwilligen holds the current position of senior expert in nautical research at Flanders Hydraulics Research. He is experienced with simulation studies, nautical accessibility studies and full scale measurements. 
He is member of the PIANC MarCom WG 171 on Ship Handling Simulation Dedicated to Channel and Harbour Design.

Guillaume Delefortrie, $\mathrm{PhD}$, naval architect, is expert nautical researcher at Flanders Hydraulics Research and visiting professor at Ghent University. He is in charge of the research in the Towing Tank for Manoeuvres in Confined Water and the development of mathematical models based on model tests. He has been secretary of the $27_{\text {th }}$ and 28th ITTC Manoeuvring Committee and is chairman of the 29th ITTC Manoeuvring Committee. 